\title{
Revue critique de fixxion française \\ XXI contemporaine
}

Statut du personnage dans la fiction contemporaine

\section{Le personnage à initiales}

Nouveaux usages d'une figure narrative singulière

\section{Aurélien d'Avout}

\section{CpenEdition}

Journals

Édition électronique

URL : https://journals.openedition.org/fixxion/651

DOI : 10.4000/fixxion.651

ISSN : 2295-9106

Éditeur

Ghent University

Référence électronique

Aurélien d'Avout, "Le personnage à initiales », Revue critique de fixxion française contemporaine [En

ligne], 23 | 2021, mis en ligne le 15 décembre 2021, consulté le 17 février 2022. URL : http://

journals.openedition.org/fixxion/651; DOI : https://doi.org/10.4000/fixxion.651

Ce document a été généré automatiquement le 17 février 2022.

\section{(c) (†) $\ominus$}

Les contenus de la Revue critique de fixxion française contemporaine sont mis à disposition selon les termes de la licence Creative Commons Attribution - Pas d'Utilisation Commerciale - Pas de Modification 4.0 International. 


\title{
Le personnage à initiales
}

\author{
Nouveaux usages d'une figure narrative singulière
}

\section{Aurélien d'Avout}

1 La présente contribution vise à appréhender les modalités et les enjeux d'une pratique romanesque peu courante, consistant à substituer une ou plusieurs initiales au nom d'un personnage. Manifeste à deux moments de l'histoire littéraire - au XVIII ${ }^{e}$ siècle, avec le genre du roman à clés, et au $\mathrm{xx}^{\mathrm{e}}$ siècle, avec la théorie du Nouveau Roman -, cet abrègement onomastique fait l'objet d'un réinvestissement de la part de la fiction contemporaine, qui accroît l'étendue de ses sens et de ses fonctions.

Discrète, ténue, l'initiale constitue une lorgnette utile par laquelle appréhender un plus vaste phénomène : la réappropriation de la notion de personnage et de ses potentialités narratives. Car les auteurs contemporains, à la différence de la génération du Nouveau Roman, réhabilitent cette catégorie par cela même qui semblait l'exclure: l'emploi d'une "onomastique parcellaire" impliquant différents types de troncatures du nom, au premier rang desquelles la réduction à l'initiale.

3 Pour en cerner les modalités et la portée profonde, l'adoption d'une focale large, et non d'une perspective monographique, apparaît plus adéquate. Cette approche globale ne se fonde pas moins sur des études de cas précises, dont les œuvres de Jean-Benoît Puech, Annie Ernaux, Delphine de Vigan, Alice Zeniter, ou encore celles de MarieHélène Lafon et Jean-Yves Laurichesse fournissent la matière. L'enjeu tient à dresser un panorama critique du procédé que nous nous proposons d'appeler l'initialisme ${ }^{2}$, c'est-àdire l'acte d'user d'une initiale en lieu et place d'un nom d'auteur, d'un nom de lieu ou, pour le cas qui nous intéresse, d'un nom de personnage.

4 Afin d'évaluer la manière dont la fiction contemporaine se saisit de l'initialisme, on évaluera l'inversion de sa valeur depuis l'époque du Nouveau Roman, avant d'appréhender le phénomène comme un opérateur de fictionnalisation du récit et de singularisation du sujet. L'intérêt de l'initialisme se fonde enfin sur son potentiel sémantique et sa portée spéculative inédite. 


\section{De l'initiale privative à l'initiale constitutive}

5 Le corps de doctrine élaboré par les théoriciens du Nouveau Roman dévalorise le personnage, perçu au même titre que l'intrigue comme un constituant obsolète du récit. Les positions avant-gardistes défendues par Nathalie Sarraute et Alain RobbeGrillet dans les années 1950 prônent une conception moderne de l'individualité, renvoyant au passé le personnage issu du roman réaliste. Celui-ci se voit retirer son cortège d'attributs, jugés arbitraires, et même jusqu'à son nom - lequel constitue la première cible identifiée dans L'ère du soupçon de Sarraute (1956). Arracher le sujet à sa gangue onomastique vise à ne pas figer le faisceau de sentiments qu'il incarne derrière une identité factice. L'insistance sur l'état civil et les index-annuaires sans fin générés par Balzac dans La comédie humaine sont érigés au rang d'anti-modèle. Proférée par les nouveaux romanciers, la mise au ban du nom sera reprise par une partie de la critique structuraliste; en 1970, Roland Barthes déclare dans $S / Z$ : “Ce qui est caduc aujourd'hui dans le roman, ce n'est pas le romanesque, c'est le personnage; ce qui ne peut être écrit, c'est le Nom Propre"3.

6 Parmi les différentes stratégies de sape onomastique, le recours à des appellatifs génériques ou l'usage de pronoms sans antécédent sont fréquents. Dans Tropismes de Sarraute, qui connaît un succès critique lors de sa réédition en 1957 chez Minuit, les personnages sont désignés par la troisième personne du pluriel ou par un lien de parenté, sans davantage de précision; le personnel romanesque du Dépeupleur de Beckett (1970) apparaît exclusivement sous la forme de périphrases, tandis que RobbeGrillet anonymise les personnages de Dans le labyrinthe (1959) en les définissant a minima par des catégories (la jeune femme, l'enfant) pouvant du reste se révéler trompeuses (le soldat devient "le pseudo-lieutenant", l'infirme le "faux invalide").

7 Si l'attribution d'un nom est conservée, sa matière sera volontiers réduite à un surnom, un prénom, ou, en l'occurrence, une initiale. Avec ses romans Le procès (1925) et Le château (1926) qui relatent les aventures respectives de Joseph K. et de K., Kafka s'impose comme la figure tutélaire de l'initialisme moderne et joue un rôle de premier plan parmi les nouveaux romanciers. Les textes de Robbe-Grillet, tels que le ciné-roman L'année dernière à Marienbad (1961) ou La reprise (2001), reprennent à leur compte l'“enveloppe légère", "le plus mince des supports"4 que constitue l'initiale. Francine Dugast-Portes commente en ces termes la désignation tronquée de la femme du narrateur dans La jalousie (1957) : “A... est aussi énigmatique que cette lettre seule avec ses points de suspension, réduite en quelque sorte définitivement à un début qu'aucun Z... ne viendra jamais achever; c'est une donnée abstraite qui peut faire venir à l'esprit le Soit initial du raisonnement mathématique" ${ }^{5}$. L'initiale est déceptive : elle apparaît comme un commencement et se révèle être une fin. Au vrai, le lecteur n'a plus affaire à une initiale mais à un signe alphabétique ne renvoyant à rien d'autre que lui-même. Choisi comme par défaut, il n'ébauche les contours, n'esquisse le périmètre d'aucune psyché, réduisant au contraire le personnage à un paramètre du récit. La dimension fictionnelle de l'être de papier est supplantée par sa valeur fonctionnelle, à l'instar des quatre personnages de la pièce de Sarraute, Pour un oui ou pour un non (1982), qui répondent aux noms - mais ce n'en sont déjà plus - de H1, H2, H3 et F. L'emploi d'initiales suspend les effets de la mimésis et contrecarre la tentation projective du lecteur. 
8 À rebours de la conception privative de l'initiale défendue par les théoriciens du Nouveau Roman, les auteurs contemporains lui accordent une valeur constitutive. Les initiales n'engagent plus une ostracisation du personnage mais se trouvent réinvesties d'un potentiel romanesque. Elles entrent ce faisant dans le jeu des points de vue et des discours intérieurs assumés par les personnages. Significative est à cet égard la scène de première rencontre entre Paul Lachalme et G. Léoty, dans Histoire du fils de MarieHélène Lafon (2020). Interne dans un lycée de province, Paul tombe malade et, conduit à l'infirmerie, apprend que l'ancienne infirmière a été remplacée :

Mademoiselle G. Léoty lui succédait. Le nom, brodé sur la blouse, plut à Paul, même si l'initiale l'inquiétait un peu. Georgette, Gisèle, Gertrude, Gilberte, Ginette, il pataugeait dans le marigot des prénoms, mais le nom avait de l'élan, de la tenue, et vibrait d'une onction élégante qui faisait image. Fiévreux et languissant, il pensa à des navires, à des envols de grives aussi, aux premiers matins mordus d'automne, quand s'ouvre le faste temps de la chasse. ${ }^{6}$

9 "Patauger dans le marigot des prénoms": l'activité de rêverie onomastique du personnage confère à l'initiale une consistance narrative au lieu de la réduire au degré zéro du nom (comme le symbolise, par sa typographie même, la figure de O. dans Les géorgiques de Claude Simon). On trouve le même cas de figure dans le roman d'Alice Zeniter, paru la même année que celui de Marie-Hélène Lafon, Comme un empire dans un empire (2020). Après avoir rencontré L au cours d'une soirée, Antoine fait "défiler sur son souvenir une succession de prénoms dont aucun ne convenait : Leïla, Louisa, Laure, Leslie, Lara, Laetitia..."'. Dans ces récits, la réduction du nom devient le point de départ d'un déploiement de l'imaginaire et d'un travail de glose rappelant ceux auxquels s'adonne Frank Flannagan dans l'une des scènes du film Ariane de Billy Wilder (1957), où le millionnaire multiplie les prénoms pour deviner l'identité d'Ariane - qui se dérobe obstinément à celui dont elle souhaite attiser le désir - à partir de l'initiale $\mathrm{A}$ figurant sur son sac à main.

L'initiale octroie d'autant plus de consistance au personnage qu'elle entre en cohérence avec son statut, professionnel ou familial, au sein de l'intrigue. Dans le roman d'Alice Zeniter, L est une hackeuse qui cherche à préserver son anonymat à tout prix, au point de délaisser son véritable prénom. Dans D'après une histoire vraie, de Delphine de Vigan (2015), la même lettre L. désigne une écrivaine de l'ombre formant aussi la principale suspecte des lettres anonymes que la narratrice reçoit à son domicile. Dans Le remplaçant d'Agnès Desarthe (2009), les initiales du grand-père de substitution de la narratrice, B.B.B., ne suggèrent pas tant l'absence du véritable aïeul déporté à Auschwitz, qu'elles ne révèlent un attachement affectif fondé sur un triple surnom (Bouz, Boris, Baruch).

\section{Teneur fictionnelle de l'initialisme}

11 À la faveur d'une inversion de polarité, les initiales ne semblent plus dirigées contre le personnage romanesque, dont la teneur fictionnelle reste garantie. Tout se passe comme si les auteurs contemporains faisaient du Nouveau Roman une parenthèse et renouaient en partie avec la tradition du roman $\mathrm{du}_{\mathrm{XVIII}}{ }^{\mathrm{e}}$ siècle, dans lequel abondent les comtes de V..., les marquises de $\mathrm{N}^{*}$, les ducs de $\mathrm{P}$. - les signes typographiques complémentaires varient en fonction des textes et parfois de leurs différentes éditions. Les initiales ne sont pas nécessairement liées au roman à clés, comme dans les œuvres de Crébillon fils où elles visent simplement à donner une aura 
de mystère à ceux qui les portent. Mais lorsqu'elles le sont, les personnages qui les portent ont beau apparaître comme les doubles anonymisés de personnes réelles, ils existent avant tout en tant que personnages fictifs. L'abrègement du nom enclenche dans ce cas une logique d'"accréditation de la fiction"s: le texte s'autodésigne comme roman à clés et fait valoir ses prérogatives génériques. De fait, les initiales exhibent leur fonction de paravent onomastique; en tant que camouflage ostentatoire, elles apparaissent comme la scintillante serrure du roman à clés, là où le genre utilise plus ordinairement un nom propre complet - risquant de passer inaperçu pour le lecteur non avisé qui ne saurait faire le lien avec la cible visée.

Dans la fiction contemporaine, l'initiale retrouve un tel potentiel fictionnalisant, les initiales contribuant à la métamorphose des personnes en personnages. Qui reconnaîtrait spontanément derrière Reeves $\mathrm{C}$., dans Les évangiles du crime de Linda Lê (2007), la figure de Reeves McCullers, l'époux de la romancière américaine Carson McCullers? Il arrive également que les initiales suscitent de la part du lecteur un décodage aisé, moins retors que certains des cryptages du roman à clés. Dans Place Monge de Jean-Yves Laurichesse (2008), le paratexte conditionne l'identification du protagoniste Jean L. à un membre de la famille de l'auteur, compte tenu de la lettre qu'il partage avec le patronyme figurant en couverture (si l'auteur avait usé d'un pseudonyme, le rapprochement n'aurait bien entendu pas été aussi évident). L'une des épigraphes du texte rend l'usage de l'initiale d'autant plus cohérent; il s'agit de celle tirée du Jardin des Plantes (1997), œuvre de Claude Simon connue pour contenir de nombreux passages sous forme d'entretiens fictifs entre un journaliste et un écrivain nommé S., dont l'expérience guerrière renvoie au vécu de l'auteur. Mais dans Place Monge, la reconnaissance de filiation n'annule pas pour autant la procédure de fictionnalisation mise en œuvre par l'initialisme, qui suscite un "jeu de desserrement du lien avec le réel"' favorisant l'imaginaire.

13 L'assimilation du personnage à initiales à une personne dont l'existence est attestée s'opère de manière plus nette encore pour $\mathrm{R}$, renvoyant à Jean-Jacques Rousseau, dans l'ouvrage "éponyme" de Céline Minard, R. (2006). Dans Louis-René des Forêts, roman de Jean-Benoit Puech (2000), le nom «LRF » attribué au personnage est conforme à celui de l'écrivain réel. Pourtant, le titre rhématique de l'œuvre invite à voir dans la réduction aux initiales un possible leurre, faisant de l'être de papier un double différencié. L'allégation d'identité qu'entraîne un initialisme transparent ne dérobe donc jamais complètement au personnage sa teneur fictionnelle.

\section{L'initiale, point de fuite du sujet}

Loin de porter atteinte à l'intégrité du personnage, les nouveaux usages de l'initialisme symbolisent souvent un trait définitoire de sa personnalité et tout particulièrement son "désir de disparaitre", dans lequel Dominique Rabaté discerne un thème majeur du roman contemporain ${ }^{10}$. La troncature du nom métaphorise la dérobade du sujet.

Dans le roman de Zeniter, L considère dès l'adolescence le "labyrinthe insoupçonné" $(C: 22)$ du codage informatique comme une échappatoire à son quotidien. Ce qu'elle nomme le "dedans" (le monde virtuel) forme pour elle un espace de construction de soi, là où elle n'évolue dans le "dehors" (le monde réel) qu'avec circonspection. Le choix du nom minimal L, au détriment d'un patronyme d'autant plus pénible à porter (“Aucun professeur ni employeur n'avait réussi à en prononcer l'amas de consonnes 
sans trébucher à la première lecture" $(c: 26))$ qu'il redouble l'absence de son père, constitue une première forme de rupture avec le monde social. L'inscription dans une communauté d'internautes est l'occasion d'une émancipation salutaire pour cette "fille $\mathrm{du}$ dehors dont tout le physique criait qu'il voulait entrer et disparaitre au-dedans" ( $C: 26)$; à l'âge adulte, $\mathrm{L}$ en est toutefois réduite à une conduite d'effacement excessive, par suite de l'incarcération de son compagnon, accusé d'avoir piraté une société de surveillance. La judiciarisation du web terrorise l'héroïne qui éprouve le sentiment constant d'être traquée. Évoluant auparavant de manière libre sur le dark web, elle doit désormais en "occuper les égouts, les terrains vagues, les ruines" $(c: 310)$, en se gardant de laisser la moindre trace.

Un vœu d'évanouissement analogue caractérise G. Léoty, la mère d'André dans Histoire $d u$ fils. Celle-ci se défausse dès l'origine de ses responsabilités maternelles en confiant son fils à sa sœur Hélène, restée à Figeac dans le Lot, et en ne lui rendant visite, depuis Paris, que deux fois par an. Son absence est à peine compensée par "de curieuses lettres évasives et très météorologiques" ( $H: 153)$, qui la maintiennent dans une forme de semi-disparition. En vérité, cette figure parentale à éclipses se définit moins par sa propre disparition que par ce qu'elle aura fait disparaître, dissimulant au père la naissance de son fils et au fils l'identité de son père. Cette disparition réciproque ne passe pas inaperçue aux yeux d'André qui, dès l'âge de dix ans et fort de ses nouvelles leçons d'écolier, se formule à lui-même : "À père inconnu, fils inconnu. Ce père et lui auraient en commun un adjectif de trois syllabes dont la première est un préfixe de sens négatif et les deux suivantes un participe passé" $(H: 61)$. Hélas, c'est en vain que le fils tâche par ses propres moyens de combler le "trou du père", de donner corps à une instance qui "faisait défaut en coulisses, creusait un vide" (H:109-110). Sa petite "affaire grammaticale" ( $H: 61)$ ne s'édifie malheureusement pour lui que sur des sables mouvants. Gabrielle (tel est le prénom qui se cachait derrière l'initiale) révèlera tardivement son secret à André, à la manière d'une "grenade dégoupillée à la table même des noces de son fils" ( $H$ : 135). Lorsqu'André tâchera enfin de s'entretenir avec son père, il n'aura avec lui qu'un rendez-vous manqué.

Le lien entre disparition et réduction à l'initiale est porté à son comble dans Les évangiles du crime, dont trois des quatre nouvelles s'organisent autour d'un suicide: Reeves $C$. est retrouvé mort dans un hôtel parisien, rongé par l'échec de sa vocation d'écrivain ; le Professeur T. se pend dans la cave de son immeuble, tandis que Klara W. se jette du haut d'un immeuble de La Défense. Les disparitions brutales de ces personnages sont figurées par l'ensevelissement avant-coureur de leurs patronymes.

\section{Densité sémantique et relance de l'interprétation}

18 Là où l'initiale pouvait paraître arbitraire (dans le roman à clés) ou privée de vocation symbolique apparente (dans le Nouveau Roman), elle apparaît investie d'un rayonnement sémantique neuf. Malgré son modeste volume, elle est capable de convoquer un réseau étendu de signifiants. Ceci est d'autant plus vrai lorsqu'elle s'associe à la mention d'une fonction ou d'un prénom : L'abbé $C$ de Georges Bataille (1950) ou le personnage Bea B. dans La guerre de Le Clézio (1970) renvoient respectivement à la naissance de l'alphabet (la suite logique A, B, C) et à l'apprentissage de la langue (le b.a ba d'une parole à énoncer). Quelques études de cas contemporains permettent d'appréhender plus finement cette dynamique. 
19 L'apprentissage du roman, de Jean-Benoît Puech (1993), se présente comme l'édition critique du journal intime de Benjamin Jordane, jeune écrivain qui noue avec PierreAlain Delancourt, son aîné, une amitié intellectuelle faite de savants échanges sur la littérature et sur ses silences. L'écrivain confirmé, qui n'a pas publié depuis une dizaine d'années, est désigné dans ce roman sous différentes appellations : son nom complet apparaît au début du texte, avant d'être relayé par différentes formes abrégées, "P.-A. Delancourt", "Pierre-Alain", "Delancourt". Le nom se stabilise rapidement sous la tournure plus laconique "PA", qui éclipse peu à peu tous les autres. Certes, l'abrègement onomastique s'accorde avec le genre même du journal intime. Par commodité, les diaristes utilisent régulièrement ce raccourci pour mentionner leurs proches, accélérant ainsi leur rythme d'écriture. Dans Le mausolée des amants (son journal tenu entre 1976 et 1991, et paru de manière posthume en 2001), Hervé Guibert utilise l'initialisme pour désigner bon nombre de ses relations, son cortège d'amis et d'amants : T. pour Thiery, le plus important, mais aussi M., G., B., Z., B.F., J.M., François P., etc. Dans le Journal d'André Gide, le dispositif connaît une légère variante à l'endroit de Madeleine, l'épouse de l'écrivain, qui n'est pas désignée par la lettre M mais par son équivalent sonore "Em." (renvoyant au prénom fictif Emmanuelle, sans doute choisi à cause de son sens étymologique).

Dans l'œuvre de Jean-Benoît Puech, le raccourci d'écriture ne fait pas que témoigner d'une proximité affective. En raison de leurs majuscules, les initiales PA monumentalisent le nom et traduisent en termes graphiques la fascination de Jordane pour l'écrivain. Phonétiquement, ces deux lettres suscitent une double interprétation : on entend à travers elles la première partie de l'appellatif “papa", en écho à la manière dont Jordane considère Delancourt (rappelant à s'y méprendre Louis-René des Forêts) comme un père spirituel. L'une des dernières notes de bas de page du livre ne précise-telle pas que "les initiales du prénom de Delancourt" sont "idéales pour symboliser la victime ou le bénéficiaire d'un transfert filial" 11 ? PA fait également entendre l'adverbe de négation "pas", symbolisant le retrait de l'auteur de la vie littéraire. La toute première occurrence de PA dans l'ouvrage est d'ailleurs immédiatement suivie par une inflation du mot. Après avoir demandé à Delancourt s'il a pris connaissance de l'un de ses courts récits publié récemment, Jordane note : “[I]l me répond que non! avec un tel accent de sincérité que je n'en déduis pas qu'il ne m'en parle pas parce qu'il ne l'aime pas! Il est vrai que mes deux pages ne sont signées que de mes initiales" ( $A: 37$, nous soulignons). L'adverbe de négation est en réalité symptomatique d'une vision de la littérature idéalisée par Jordane, qui admire chez Delancourt ce qu'il considère comme un adieu au langage. Aux yeux du jeune écrivain, l'absence de publication n'a rien d'un échec, d'une faille ou d'un épuisement ; elle s'apparente bien plutôt à une "réalisation de ce que l'œuvre annonçait", à une forme paradoxale de "passage à l'acte"12 (A:129) l'expression étant elle aussi réductible aux initiales PA - marquant l'indépendance de l'auteur à l'égard des servitudes de la langue et du regard d'autrui, du "monde des mots et de [s]es semblables" ( $A: 138)$. Le lecteur apprendra pourtant que cette thèse n'est guère partagée par Delancourt, pour qui le silence ne constitue pas un acte significatif, et encore moins le "refus de l'expression" ( $A: 82)$ prôné par Jordane, mais tient à des raisons contingentes. Lui prêter une valeur littéraire, comme les critiques le font pour Rimbaud, s'apparente à une vue de l'esprit, une assertion arbitraire, le "comble de la déformation" ( $\mathrm{A}: 127)$. Malgré tout, Jordane peine à se détacher de ses propres conceptions et continue à utiliser les initiales jusqu'à la fin du livre, avant de reconnaître in extremis avoir eu "terriblement tort de [s]'obstiner à réduire" le silence 
de PA “à celui qui [l]'obsédait" ( $A: 232)$. Comme Alexandre Gefen l'a mis en évidence, Jean-Benoît Puech réfute, par l'entremise de ses personnages, le "mensonge blanchotien d'un écrivain hors le monde et désincarné"13. Mais cette insistance sur le silence ne signifie-t-elle pas également le renoncement de Puech lui-même à la figure de l'auteur démiurgique, lui qui affecte de jouer le rôle plus modeste d'éditeur et habite sa création à l'ombre des seules initiales “J.-B.P.", présentes dans la préface et la postface de l'ouvrage? En tout état de cause, l'écrivain Delancourt finit par quitter la sphère du "pas", l'initialisme PA disparaissant de manière symptomatique au profit d'un ultime nom de baptême, Paul-André Montmurel, qui relance le vertige des jeux identitaires.

21 L'initiale L, à la surface de laquelle affleure le pronom "elle", possède elle aussi une valeur sémantique dense, susceptible d'interprétations divergentes selon les ouvrages où elle est employée. Dans D'après une histoire vraie, L. représente pour Delphine l'idéal de la féminité, dont témoignent sa sophistication vestimentaire, son aisance en société et son "mystérieux mélange de mouvement et d'apparat" ${ }^{14}$. Elle fait progressivement l'objet d'une "cristallisation spectaculaire" $(D: 144)$ de la part de la narratrice - qui se dévalorise de manière inversement proportionnelle -, tout comme Jordane, "pren[ant] les Grands Écrivains pour des Saints" (A:29), idéalisait PA. Le Grand Autre semble ne jamais mieux s'énoncer que par le détour de ses initiales.

Chez Alice Zeniter, le sémantisme de l'initiale L diffère, soulignant cette fois le féminisme d'un personnage qui refuse l'héritage du nom du père et décide par ailleurs de mettre son expertise informatique au service de femmes subissant la violence de leurs conjoints. Sans aller jusqu'au dispositif adopté par Anne F. Garréta dans Sphinx (1986), où l'emploi de l'initiale rend indéterminable l'identité sexuelle de $\mathrm{A}^{* * *}$, Zeniter propose une déconstruction des normes de genre imposées en particulier par le système marchand. La brève expérience professionnelle de L dans la boutique d'une firme célèbre souligne l'incompatibilité du personnage aux codes promus par la marque et permet de dénoncer leur caractère oppressif :

Si être une femme voulait dire ressembler à ses collègues, alors L préférait qu'on raye tout de suite son nom de la liste, elle n'en serait jamais. Ce qu'elles appelaient "féminité", L l'appelait temps et argent perdus et elle aurait pu s'en moquer si cette féminité-là n'avait pas été prosélyte (L pensait "colonisatrice"), si on lui avait foutu la paix avec sa propre manière d'être femme. $(C: 259)$

S'il est vrai qu'on ne peut jamais s'assurer de l'intention d'un auteur et si la motivation sémantique de l'initiale n'est pas nécessairement de son fait, l'interprétation du lecteur semble du moins la garantir à chaque coup.

\section{Spéculation et spécularité}

Les initialismes favorisent l'activité spéculative, qu'ils multiplient les jeux de décodage et de reconnaissance ou poussent la réflexion du côté de la nature du roman et de ses actants. Les fictions autobiographiques d'Annie Ernaux reconduisent autrement la portée épistémique de l'initialisme, en l'utilisant non pas comme un outil de spéculation métaromanesque mais comme un instrument d'investigation du réel. Dans L'occupation (2002), les initiales semblent d'abord renouer avec l'acte de cryptage propre aux romans à clés. Elles apparaissent tributaires d'un "système de décalage" généralisé, "employé par discrétion, ou quelque motivation plus ou moins 
consciente"15. Annie Ernaux met le lecteur au défi d'élucider l'identité des personnages mis en scène : l'amant de la narratrice, W. ; l'amie de son fils, F.; et surtout la nouvelle compagne de l'amant, une éventuelle Dominique L. Le recours aux initiales entre ici en cohérence avec la jalousie de la narratrice et avec l'enquête qu'elle conduit sur l'identité de sa rivale, dont le nom reste "un trou, un vide, autour duquel [elle] tourn[e]" en vain malgré l'“assemblage effréné des signes" ( $0: 887$ et 892). Pour autant, Annie Ernaux contourne le simple jeu de piste, déclarant au lecteur "décline[r] d'avance la sollicitude d'éventuels informateurs" $(0: 892)$. Derrière la boutade, l'enjeu tient à préserver la visée aléthique du texte. En brouillant les paramètres de l'histoire racontée - les noms de lieux subissent d'ailleurs le même sort que ceux des personnages -, les initiales lui confèrent une portée plus générale. La narratrice reconnaît qu'elle ne souhaite pas tant narrer une crise personnelle qu'analyser la jalousie en tant que telle. Dans L'événement (2000), les noms des personnages - le docteur N., P., le petit ami de la narratrice, ou O., sa voisine de chambre - se trouvent pareillement anonymisés, focalisant l'attention du lecteur sur le drame de l'avortement avant sa légalisation en France. Dans chaque cas, réduire la matière onomastique équivaut à désenclaver l'expérience et à convertir sa singularité en généricité. Ce faisant, les initiales fictionnalisent noms et lieux tout en continuant de faire signe vers le réel, de sorte que la pratique narrative d'Ernaux "n'est pas un épanchement, mais une exploration de la réalité, accompagnée de la volonté, sans doute, que le lecteur ne s'irréalise pas dans le texte, se questionne au contraire sur son propre parcours, mette au jour des sentiments enfouis"16.

Un tel projet vaut en particulier pour les récits plus directement liés à l'histoire familiale, comme La place (1983), Une femme (1988) ou La honte (1997), dans lesquels les initiales employées demeurent conformes aux noms réels, à l'instar de "la petite D." renvoyant au nom de jeune fille de l'autrice, Annie Duchesne. Dans cette configuration, l'initialisme se conçoit comme une technique d'anonymisation empruntée aux sciences humaines et à l'entretien sociologique, ainsi qu'Isabelle Charpentier le soutient ${ }^{17}$. Tirant l'introspection du côté de l'“extrospection"18, Annie Ernaux abrège les noms pour mieux objectiver les mécanismes sociaux. Les initiales, par leur tournure impersonnelle, incitent donc le lecteur à reconnaître en elles moins des personnages que des points, des postures et des positions dans le champ social. Elles sont un outil privilégié de la "langue d'analyse" ${ }^{19}$ forgée par l'autrice et relèvent d'une recherche formelle et stylistique plus vaste. Mise à plat du nom propre, l'initialisme s'impose comme l'une des modalités de l'“écriture plate" choisie par Ernaux pour restituer le milieu humble dans lequel elle a grandi.

Dans D'après une histoire vraie de Delphine de Vigan, l'initialisme n'a pas tant vocation à dissimuler une identité civile qu'à interroger l'identité du texte, la fabrique du personnage, le statut de la fiction. L'argument du livre est le suivant: après une première rencontre dans une soirée parisienne, L. s'immisce par approches successives dans la vie de Delphine, la narratrice, au point de phagocyter son existence. Celle-ci plonge bientôt dans une totale dépendance à l'égard de cette amitié nouvelle qui intervient dans un temps de doute personnel et d'éloignement familial. Cette relation d'emprise bascule à partir du moment où Delphine autorise L. à usurper son identité : en lui déléguant l'acte de répondre aux courriers et aux sollicitations qu'elle reçoit, puis en acceptant qu'elle se rende à sa place à une rencontre littéraire. La tension narrative du roman se résout avec un acte d'empoisonnement à la mort aux rats : le 
corps de Delphine est retrouvé dans le bas-côté d'un chemin de campagne et elle échappe à la mort de peu. Pourtant, rien ne permet d'établir qu'il s'agit là d'une tentative de meurtre. Sommée de décliner l'identité de L., Delphine n'est pas même capable de fournir une preuve objective de son existence: L. s'est arrangée pour ne jamais rencontrer l'entourage de Delphine, ne laisser derrière elle aucun effet personnel ni aucune trace écrite.

L'éventail des ressemblances entre Delphine et L., son alter ego, ne serait dès lors qu'un trompe-l'œil. La fin du roman pousse la relation de gémellité jusqu'à son paroxysme, en suggérant que les deux personnages, solubles l'un dans l'autre, n'en forment en réalité qu'un seul. Sans doute L. constitue-t-elle le double fantasmé, le "décalque aux dimensions copiées" ( $D: 131)$ de Delphine qui, mue par "l'espoir inassouvi [...] d'être quelqu'un d'autre" ( $D: 88)$, aurait créé, à la manière des enfants, une amie imaginaire. C'est ce que confirme la prise de conscience de Delphine : L. s'inspirait des ouvrages de sa bibliothèque, lors de leur cohabitation, pour se forger de toutes pièces une vie passée. Dit autrement, L. est peut-être le roman que s'est inventé Delphine, à la fois démiurge et victime, marionnettiste manipulée par sa créature. Le roman se clôt sur un jeu de miroir plus complexe que prévu: le mot "FIN" s'accompagne d'un astérisque renvoyant à la signature discrète que reconnaît utiliser $\mathrm{L}$. à la fin des livres qu'elle écrit pour d'autres (en tant que ghost writer, elle ne mentionne jamais son propre nom). Elle accomplit par finesse ce que Delphine, par épuisement, reconnaît lors d'un salon du livre ne plus être en mesure de réaliser : “je n'en peux plus d'écrire mon nom, mon nom est une imposture, une mystification" ( $D: 20)$. L'astérisque final bouleverse le statut du texte en extrayant $\mathrm{L}$. de la diégèse et en faisant d'elle l'auteur du livre que le lecteur a sous les yeux. De même qu'au "coup de gong final le récit devient contemporain de tous ses moments", suivant la formule de Paul Claudel tirée de Positions et propositions, l'ultime signe graphique de l'ouvrage fait se superposer, dans une coïncidence parfaite, la productrice du texte (Delphine de Vigan), son double autofictionnel (Delphine), et le double de son double (L.).

La première lettre anonyme du roman accusait la narratrice de se croire quitte parce que son premier livre - renvoyant à Rien ne s'oppose à la nuit (2011) - est "soi-disant un roman" au prétexte qu'elle a "changé quelques prénoms" ( $D: 45)$. Avec son nouvel ouvrage, Delphine de Vigan mène une tout autre stratégie : en choisissant la voie de l'initialisme plutôt que celle de l'onomaturgie (l'invention de noms fictifs), elle porte beaucoup plus loin la réflexion sur les frontières entre fiction et réalité, au centre des dialogues échangés entre L. et Delphine. L'initiale constitue le signe distinctif d'un questionnement sur une impossible distinction: le personnage est-il le produit de l'auteur, ou l'auteur celui de son personnage?

S'il pouvait apparaître comme une pratique un peu désuète, l'initialisme fait en réalité l'objet d'une réactualisation dans la fiction contemporaine. Les personnages à initiales ne sont certes pas légion au regard de l'immensité de la production littéraire, mais reliés les uns aux autres, ils forment une constellation significative qui interroge et met en évidence les pouvoirs de la fiction, ses mécanismes comme sa complexité. 


\section{NOTES DE FIN}

1. Nous empruntons l'expression à Frank Wagner, "Perturbations onomastiques : l'onomastique romanesque contre la mimèsis", dans Yves Baudelle (dir.), Onomastique romanesque, Paris, L'Harmattan, 2008, p. 25.

2. L'initialisme désigne à l'origine une forme de pseudonymat employé dans le monde du journalisme, où l'auteur d'un article signe ce dernier des initiales de son propre nom ou d'un nom inventé.

3. Roland Barthes, S/Z, Paris, Seuil, 1970, p. 102.

4. Nathalie Sarraute, L'ère du soupçon, Paris, Gallimard, 1987 [1956], <Folio essais>, p. 45.

5. Francine Dugast-Portes, Le Nouveau Roman: une césure dans l'histoire du récit, Paris, Nathan Université, 2001, p. 82.

6. Marie-Hélène Lafon, Histoire du fils, Paris, Buchet/Chastel, 2020, p. 37-38 (dorénavant $H$ ).

7. Alice Zeniter, Comme un empire dans un empire, Paris, Flammarion, 2020, p. 253 (dorénavant C).

8. Frank Wagner, art. cit., p. 25.

9. Entretien avec l'auteur réalisé le 23 avril 2021.

10. Voir Dominique Rabaté, Désirs de disparaître. Une traversée du roman français contemporain, Rimouski/Trois-Rivières, Tangence éditeur, 2015, <Confluences>.

11. Jean-Benoît Puech, L'apprentissage du roman, Seyssel, Champ Vallon, 1993, p. 240 (dorénavant $A$ ).

12. Ibid., p. 129.

13. Alexandre Gefen, "Benjamin Jordane, roman : jeux identitaires et aventures métatextuelles dans l'œuvre de Jean-Benoît Puech", dans Pierre Lecœur et Dominique Rabaté (dirs), Les mondes de Jean-Benoît Puech, Paris, Classiques Garnier, 2016, p. 80.

14. Delphine de Vigan, D'après une histoire vraie, Paris, Jean-Claude Lattès, p. 72 (dorénavant $D$ ).

15. Annie Ernaux, L'occupation, dans Écrire la vie, Paris, Gallimard, 2011 [2002], <Quarto Gallimard>, p. 909 (dorénavant 0).

16. Annie Ernaux, "Raisons d'écrire", dans Jacques Dubois, Pascal Durand et Yves Winkin (dirs), Le symbolique et le social : la réception internationale de Pierre Bourdieu, Liège, Presses universitaires de Liège, 2005, p. 346. Nous soulignons.

17. Isabelle Charpentier, "L'œuvre auto-sociobiogaphique d'Annie Ernaux ou les incertitudes d'une posture improbable"], COnTEXTES, $\mathrm{n}^{\circ} 1$, "Discours en contexte", 2006, disponible sur <https://journals.openedition.org/contextes/74> (page consultée le 15 mai 2021).

18. Voir Danilo Martuccelli, La société singulariste, Paris, Armand Colin, 2010.

19. Annie Ernaux, Retour à Yvetot, Paris, Éd. du Mauconduit, 2013, p. 33.

\section{RÉSUMÉS}

La présente contribution vise à appréhender les modalités et les enjeux d'une pratique romanesque relativement rare, consistant à substituer une ou plusieurs initiales au nom d'un personnage. Manifeste à deux moments de l'histoire littéraire - au XVIII ${ }^{\mathrm{e}}$ siècle avec le genre du roman à clés et au $\mathrm{XX}^{\mathrm{e}}$ siècle avec le Nouveau Roman -, cette pratique onomastique est réinvestie par la fiction contemporaine, qui en accroît l'étendue des sens et des fonctions. Entraînant une singularisation paradoxale du sujet, des jeux sémantiques inédits, une relance de l'interprétation, un tel usage parcellaire du nom soulève des enjeux d'ordre théorique sur cette composante essentielle du récit qu'est le personnage. L'article en interroge la diversité et la complexité des formes à partir d'une série d'études de cas - dont les principales correspondent aux œuvres de 
Jean-Benoît Puech, Annie Ernaux, Alice Zeniter, Delphine de Vigan ou encore Marie-Hélène Lafon et Jean-Yves Laurichesse.

INDEX

Mots-clés : onomastique, initiales, référentialité, théorie littéraire, autofiction

\section{AUTEURS}

AURÉLIEN D'AVOUT

Université de Rouen 